\title{
Changes in the gene expression of chalkbrood resistance in Apis mellifera larvae infected by Ascosphaera apis
}

\author{
Hongyi $\mathrm{NIE}^{1}$, Xueyan $\mathrm{WANG}^{1}$, Shupeng $\mathrm{Xu}^{1}$, Yan $\mathrm{GAO}^{1}$, Yan $\mathrm{LIN}^{1}$, Yanan $\mathrm{ZHU}^{1}$, \\ Donglin $\mathrm{YANG}^{2}$, Zhiguo $\mathrm{Li}^{1}$, Songkun $\mathrm{Su}^{1}$ \\ ${ }^{1}$ College of Animal Sciences (College of Bee Science), Fujian Agriculture and Forestry University, Fuzhou 350002 China \\ ${ }^{2}$ Chongqing Key Laboratory of Kinase Modulators as Innovative Medicine, IATTI, Chongqing University of Arts and \\ Sciences, Chongqing 402160 China
}

Received 4 May 2019 - Revised 6 September 2019 - Accepted 9 October 2019

\begin{abstract}
Chalkbrood is a type of common fungal disease that affects colony health, productivity, and pollination of honey bees. In this study, resistant and susceptible larvae towards chalkbrood were initially determined by using SNP C2587245T. We compared the transcriptome of the resistant and susceptible individuals challenged with Ascosphaera apis and found that 172 and 207 genes were specially and differentially expressed in the midguts and hemolymph of the resistant larvae infected with A. apis, respectively. Besides, the immune response of honey bees infected with A. apis was investigated. Most interestingly, it was found that the antimicrobial peptide defensin 1 was significantly and exclusively upregulated in the hemolymph of the resistant individuals infected with A. apis. This comprehensive transcriptome analysis substantially helped enhance our understanding of the mechanisms of chalkbrood resistance and the corresponding gene expression during infection with A. apis .
\end{abstract}

chalkbrood resistance / RNA-seq / midguts / hemolymph / antimicrobial peptides

\section{INTRODUCTION}

Chalkbrood is a kind of honey bee brood disease caused by the heterothallic fungus Ascosphaera apis, affecting colony health, productivity, and pollination of agricultural crops. This disease currently exists worldwide; thus, it is considered as one of the most serious health problems of honey bees (Aronstein and Murray 2010). A. apis was primarily detected in Apis

Electronic supplementary material The online version of this article (https://doi.org/10.1007/s13592-019-00702-y) contains supplementary material, which is available to authorized users.

Corresponding author: S. Su, susongkun@zju.edu.cn Hongyi Nie and Xueyan Wang contributed equally to this work.

Manuscript editor: Klaus Hartfelder mellifera and presented in Apis cerana, Xylocopa augusti, and bumble bees (Chen et al. 2018).

Chalkbrood has been extensively studied in terms of pathogen biology and host-pathogen interactions. Many studies, including morphology (Skou 1988; Li et al. 2018), pathology (Aronstein et al. 2007; Theantana and Chantawannakul 2008), and epidemiology (Flores et al. 2005), on A. apis have been conducted. With the completion of the sequencing of $A$. apis, the comprehensive transcriptome analysis of $A$. apis was carried out, and recently 379 lncRNAs and 118 miRNAs were identified in the chalkbrood pathogen (Qin et al. 2006; Cornman et al. 2012; Guo et al. 2018a, b). When A. apis infected honey bee larvae, host transcriptional responses were investigated through cDNA-AFLP technology and transcriptome sequencing, providing a number of differentially regulated genes in response to the invasive fungal pathogen (Aronstein et al. 
2010; Chen et al. 2017). Several alternative strategies, including testing antifungal agents and natural plant products, improving management and sanitation practices, improved genetic stock and use of Gamma irradiation, have been developed and implemented to control chalkbrood disease (Aronstein and Murray 2010). Until now, neither chemicals nor natural plant-derived products have been approved for the control of chalkbrood in bee colonies. Therefore, selecting and breeding chalkbrood resistance species can be the most effective defensive strategy. Based on our previous studies, it was found that the $\mathrm{C}$ allele at SNP C2587245T was strongly associated with chalkbrood resistance (Liu et al. 2016). Since infected larvae usually die long before the appearance of visible signs of the disease, this SNP may serve as a useful marker for the selection of chalkbrood-resistant honey bees at an early stage. However, the resistance mechanisms in bees remain largely unknown.

Once the fungal spores or ascospores in food are ingested by larvae, ascospores will germinate in the midguts, and fungi will invade the internal organs; consequently, the mycelia will exit the cadaver to form aerial hyphae on the skin surface (Cornman et al. 2012; Aronstein and Holloway 2013). In insects, antimicrobial peptides (AMPs), which are synthesized in the fat body and then secreted into the hemolymph, are effector molecules against pathogenic microorganisms (Lourenço et al. 2018). Hemolymph is also an innate immune system against invasive microorganisms (Vierstraete et al. 2003; Kim and Kim 2005; Chan et al. 2009). Therefore, hemolymph is suitable for evaluating the systemic response of honey bees to A. apis. Hence, the resistant and susceptible individuals were initially determined by using SNP C2587245T identified from our previous study. We also systematically compared the gene expression in the midguts and hemolymph of the resistant and susceptible larvae through RNA-seq. We focused on the unique differential expressed genes (DEGs) in the midguts and hemolymph of the resistant individuals and immune response genes associated with chalkbrood in midguts and hemolymph. These data provided new insights into the mechanisms of chalkbrood resistance and relevant gene expression during infection and also offered clues towards future molecular studies on biocontrol methods to chalkbrood disease and breeding of resistant lines.

\section{MATERIAL AND METHODS}

\subsection{Honey bee colonies}

Fengqiang No. 1 Italian honey bees (A. mellifera lingustica) with high royal jelly production were maintained at the apiaries in the (College of Animal Sciences) College of Bee Science, Fujian Agriculture and Forestry University. Three healthy colonies with no obvious symptoms of disease were used in our experiment and maintained in standard beekeeping practices. Each colony, containing a young normal egg-laying queen, had a working population of 8 frames of comb with larvae, pupae, honey, and pollen.

\subsection{Larval rearing and inoculation}

Honey bee larvae were reared in accordance with the protocols developed by Jensen et al. (Jensen et al. 2009). After the queens, together with workers in colonies, were confined in empty combs for 6 h, 2-day-old larvae were grafted into 48-well tissue culture plates with a droplet of 10 $\mu \mathrm{L}$ of diet in each well by using the Chinese grafting tool. The diet was supplied in accordance with the recipe developed by Zachary Huang (Vojvodic et al. 2011). The culture plates were kept in a $34{ }^{\circ} \mathrm{C}$ and $95-99 \%$ relative humidity incubator for 4 days in darkness. The larvae were fed once per day based on the quantities used in Jensen et al. (2009). In the treatment groups, each 3-day-old larva was fed with $10 \mu \mathrm{L}$ of contaminated diet containing $5 \times 10^{5}$ spores (Jensen et al. 2013 ), and $10 \mu \mathrm{L}$ of normal diet was then provided when the contaminated diet was fully ingested.

\subsection{Sample collection and assessment of chalkbrood resistance through SNP}

To determine the optimal day for collecting samples, we initially inoculated artificially reared 3day-old larvae with A. apis. The survival analysis following infection showed that a great number of 
larvae in the treatment group began to die on the day of the 6-day-old (Fig. S1). Hence, we collected all of the samples from 6-day-old larvae for subsequent experiments. In brief, the 6-day-old larvae reared with normal diet without $A$. apis spores were regarded as the control group, whereas 6-day-old larvae reared with diet containing A. apis spores were the treatment group. The midguts and hemolymph of the treatment and control groups were collected individually, and corresponding epidermis was also collected to determine whether the larvae were resistant. The DNA in each sample was extracted from the epidermis as a PCR template. PCR was performed to obtain DNA fragments, including SNP C2587245T, in which the C allele at SNP C2587245T was related to chalkbrood resistance (Liu et al. 2016). Thus, we sequenced the PCR product and selected the larvae with $\mathrm{C} / \mathrm{C}$ and $\mathrm{T} / \mathrm{T}$ genotypes as resistant and susceptible samples, respectively. After PCR sequencing, we collected the midguts and hemolymph tissue from the resistant and susceptible individuals. Subsequently, we obtained 24 samples (three replicate libraries for each group) for RNA sequencing on the basis of the allele at SNP C2587245T: the midguts of the C/C larvae from the control group (C_M_CC in which $\mathrm{C}$ represented the control group, $\mathrm{M}$ represented the midguts, and $\mathrm{CC}$ represented the $\mathrm{C} / \mathrm{C}$ larvae); the midguts of the $\mathrm{T} / \mathrm{T}$ larvae from the control group (C_M_TT in which TT represented the T/T larvae); the midguts of the $\mathrm{C} / \mathrm{C}$ larvae from the treatment group (M_CC in which M represented the midguts, and $\mathrm{CC}$ represented the $\mathrm{C} / \mathrm{C}$ larvae); the midguts of the T/T larvae from the treatment group (M_TT in which TT represented T/T larvae). Similarly, the hemolymph samples were also divided into four groups (C_H_CC, C_H_TT, H_CC and H_TT), in which $\mathrm{H}$ represented the hemolymph.

\subsection{Library preparation and Illumina sequencing}

The total RNA of each sample was extracted and detected as our previous methods (Nie et al. 2018). Twenty-four sequencing libraries were constructed in accordance with the procedures described previously with minor modifications (Zhang et al. 2013). In brief, the mRNAs were enriched, fragmented into short pieces (about 300 nt) and reverse transcribed to cDNAs with a random hexamer primer and M-MuLV reverse transcriptase (RNase H-). Sequencing adaptors were ligated to the cDNA fragments through PCR amplification. Sequencing analysis was performed using HiSeq X Ten (Illumina, Inc. San Diego, USA) in Novogene Bioinformatics Institute (Beijing, China), and 150-bp paired-end reads were generated. The raw data presented in this article have been deposited to NCBI Short Read Archive (http://www.ncbi.nlm.nih.gov/sra/) and are accessible through SRA accession number: SRP151761.

\subsection{RNA-seq analysis}

Clean reads were subsequently mapped to the A. mellifera genome (Amel 4.5, ftp://ftp.ncbi.nih. gov/genomes/Apis_mellifera/Assembled chromosomes/seq/) by using Hisat2 (V2.0.5). StringTie (V1.3.3b) was employed to assemble the mapped reads of each sample (Pertea et al. 2015). FeatureCounts v1.5.0-p3 was used to count the number of reads mapped to each gene, and fragments per kilobase of transcript per million fragments mapped (FPKM) was employed to quantify transcript expression (Mortazavi et al. 2008; Liao et al. 2014). In differential expression analyses, the DESeq2 R package (1.16.1) was employed to determine differential expression by using a model based on the negative binomial distribution. The read counts were adjusted by one scaling normalized factor prior to the differential gene expression analysis (Dillies et al. 2013 ). The $P$ values were adjusted using Benjamini and Hochberg's approach to control the false discovery rate. A corrected $P$ value $<0.05$ by DESeq2 was set as the threshold for significant differential expression. In addition, the functional analysis of DEGs was proceeded to GO enrichment analysis by the clusterProfiler $\mathrm{R}$ package.

\subsection{RNA-seq data validation through qRT- PCR}

Reverse transcription was performed using $1 \mu \mathrm{g}$ of total RNA and the PrimeScript RT reagent kit (RR037A, Takara). qRT-PCR was conducted using an ABI7500 real-time PCR system (Applied 
Biosystems). All of the reactions and PCR conditions were performed in accordance with our previous methods (Nie et al. 2014; Nie et al. 2018). Eleven genes were randomly chosen from DEGs to verify the RNA-seq results. The changes in the expression level determined by qRT-PCR were consistent with those of RNA-seq data (Fig. S2). Each qRT-PCR experiment was performed using three replicates. The reference gene actin (NM_001185146.1) was used as a control for normalization. Data were analyzed with the analysis of variance (ANOVA) by using GraphPad Prism 5. Differences were considered significant if $P<0.05$. The gene-specific primers for qRT$\mathrm{PCR}$ are listed in Table S1.

\section{RESULTS}

\subsection{Construction of the honey bee midguts and hemolymph libraries}

Prior to RNA extraction, larvae were sequenced to identify the resistant or susceptible larvae on the basis of the genotype of SNP C2587245T. To obtain the A. apis -affected transcriptome of larvae midguts and hemolymph, 24 total RNA samples were prepared from A. apis treated and non-treated 6-day-old larvae, followed by paired-end cDNA library construction and RNA sequencing. The statistics for all samples are summarized in Table S2. After quality control, the number of clean bases for each library was approximately $7 \mathrm{~Gb}$. Paired-end mRNA reads with $150 \mathrm{bp}$, and a Q30 percentage of 89-95\% was generated and sequentially mapped to the A. mellifera reference genome. The proportion of uniquely mapped reads in the 24 transcriptome libraries ranged from 87.5 to $92.46 \%$.

\subsection{Specific DEGs in the midguts of the resistant larvae with $A$. apis infection}

Using a significance threshold of the corrected $P$ value $<0.05$ by DESeq 2 and considering the individuals with $\mathrm{C}$ and $\mathrm{T}$ alleles at SNP C2587245T as the resistant and susceptible larvae to A. apis, respectively, we identified 298 DEGs in the midguts of the resistant larvae C_M_CC exposed to A. apis and 432
DEGs in the midguts of the susceptible larvae C_M_TT exposed to A. apis (Figure 1a). Remarkably, 172 of the 298 DEGs were unique in the midguts of resistant larvae M_CC. Of these genes, 90 were upregulated and $\overline{82}$ were downregulated in the treatment group compared with those in the control group C_M_CC (Figure 1b). Genes with FPKM in the interval 0 -1 were considered not to be expressed. Approximately $37.8 \%$ (34 genes) of the upregulated genes were not expressed $(\mathrm{FPKM}<1)$ in the midguts of the C_M_CC, and they were significantly upregulated when the larvae were treated with $A$. apis. These genes were no expression or present at very low levels in the midguts of the susceptible individuals C_M_TT, and their expression was slightly increased after exposure to A. apis and did not significantly differ from that of the control group (Table S3). Among them, three cytochrome P450 (CYP) genes, namely cytochrome P450 6A1, cytochrome P450 6AS5, and novel.10695 (containing PF00067: Cytochrome $\mathrm{P} 450$ ), were upregulated in the midgut of the resistant larvae.

\subsection{Specific DEGs in the hemolymph of the resistant larvae with $A$. apis infection}

A total of 289 genes in the C_H_CC and 498 genes in the C_H_TT had different expression patterns in the hemolymph exposed to A. apis (Figure 1c). Remarkably, 207 of the 289 genes were exclusive in the hemolymph of the resistant larvae exposed to A. apis. Of these genes, 118 were upregulated and 89 were downregulated in the treatment group compared with those in the control group C_H_CC (Figure 1d). Among the 118 upregulated genes, $46.6 \%$ (55 genes) were not expressed in the hemolymph of the C_H_CC, and they were significantly upregulated when the larvae were treated with A. apis. These genes were also not expressed or only present at low levels in the hemolymph of $\mathrm{C}_{-} \mathrm{H}_{-} \mathrm{TT}$, and their expression slightly increased after exposure to A. apis, but this increase was not significantly different from that in the control group (Table S4). The top two upregulated 

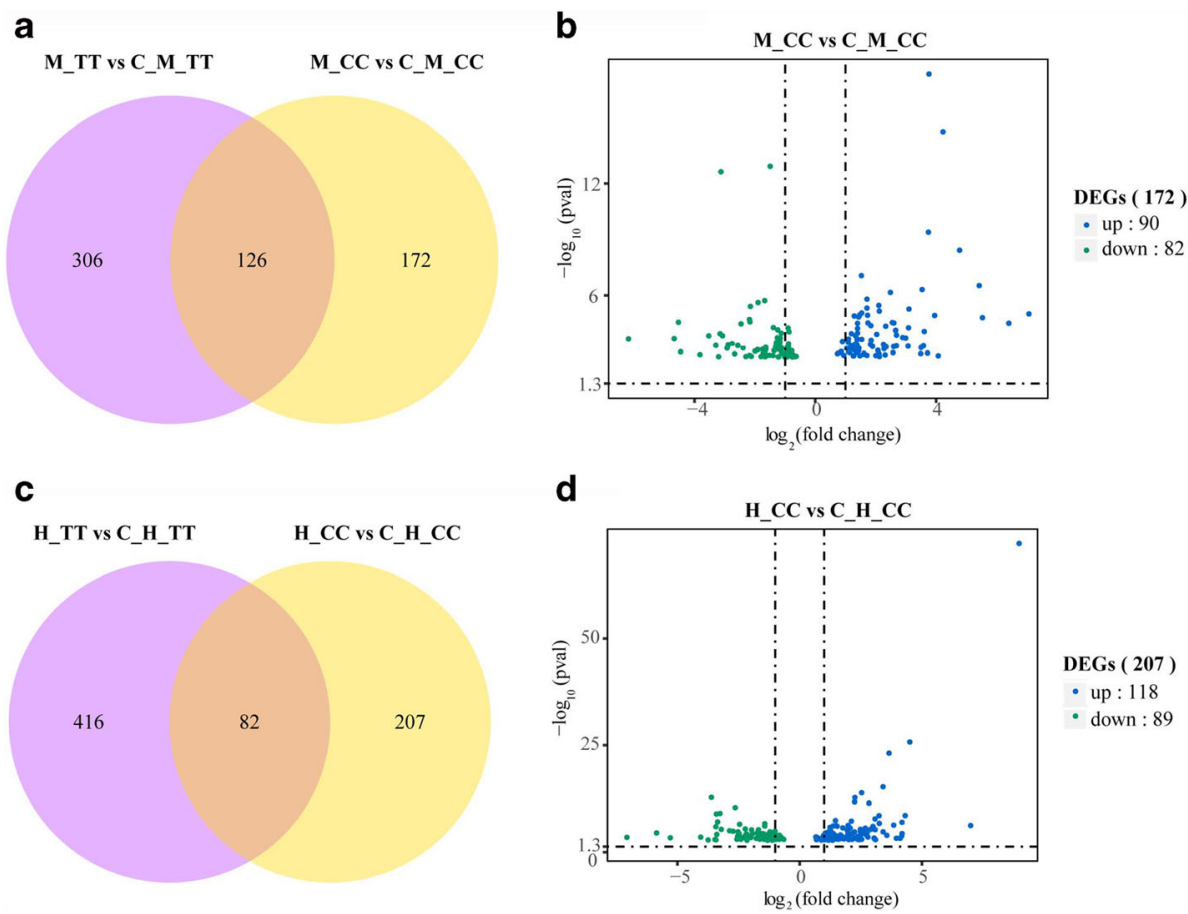

Figure 1. Similarities and differences of gene expression in the midguts/hemolymph of resistant and susceptible larvae exposed to A. apis . a Venn diagram indicates the numbers of differentially expressed genes for each context. b Difference in the regulation of DEGs in the midguts of resistant larvae. c Venn diagram indicates the numbers of differentially expressed genes for each context. d Difference in the regulation of DEGs in the hemolymph of resistant larvae.

genes were defensin 1 and cytochrome P450 $6 A 1$ in the hemolymph of the resistant larvae infected with $A$. apis. Most surprisingly, defensin 1 was highly expressed (FPKM = 940) in the hemolymph of the resistant individuals treated with $A$. apis, which was up to approximately 500 fold compared with its expression $(\mathrm{FPKM}=1.9)$ in the hemolymph of individuals C_H_CC. Moreover, the gene expression was still low $(\mathrm{FPKM}=1.9)$ in the hemolymph of susceptible larvae C_H_TT. This finding was consistent with the expression of defensin 1 in the hemolymph of the C_H_CC and was slightly increased (FPKM = $10.7)^{-}$in the hemolymph of the individuals H_TT. The expression of defensin 1 was dramatically upregulated in the hemolymph of the resistant individuals compared with that of the susceptible larvae, suggesting that defensin 1 might function in the hemolymph resistance to chalkbrood.

\subsection{Common DEGs in the midguts and hemolymph of the resistant larvae exposed to A. apis}

To investigate the common DEGs in the midguts and hemolymph of the resistant larvae exposed to A. apis, we analyzed the unique DEGs in each tissue and found that 17 genes were differentially expressed in the midguts and hemolymph (Figure 2 and Table S5). Among these genes, 9 genes, including mucin-19, yellow-e3, 5'-nucleotidase domain-containing protein 3 , cytochrome P450 6A1, and five novel genes, exhibited upregulated expressions in the midguts and hemolymph of the resistant individuals treated with A. apis. By contrast, eight genes, including myosin regulatory light chain 2, methionine sulphoxide reductase A, troponin I, anaphase-promoting complex subunit CDC26-like, uncharacterized LOC102653856, uncharacterized protein At4g17910, and another two novel genes, were downregulated. 


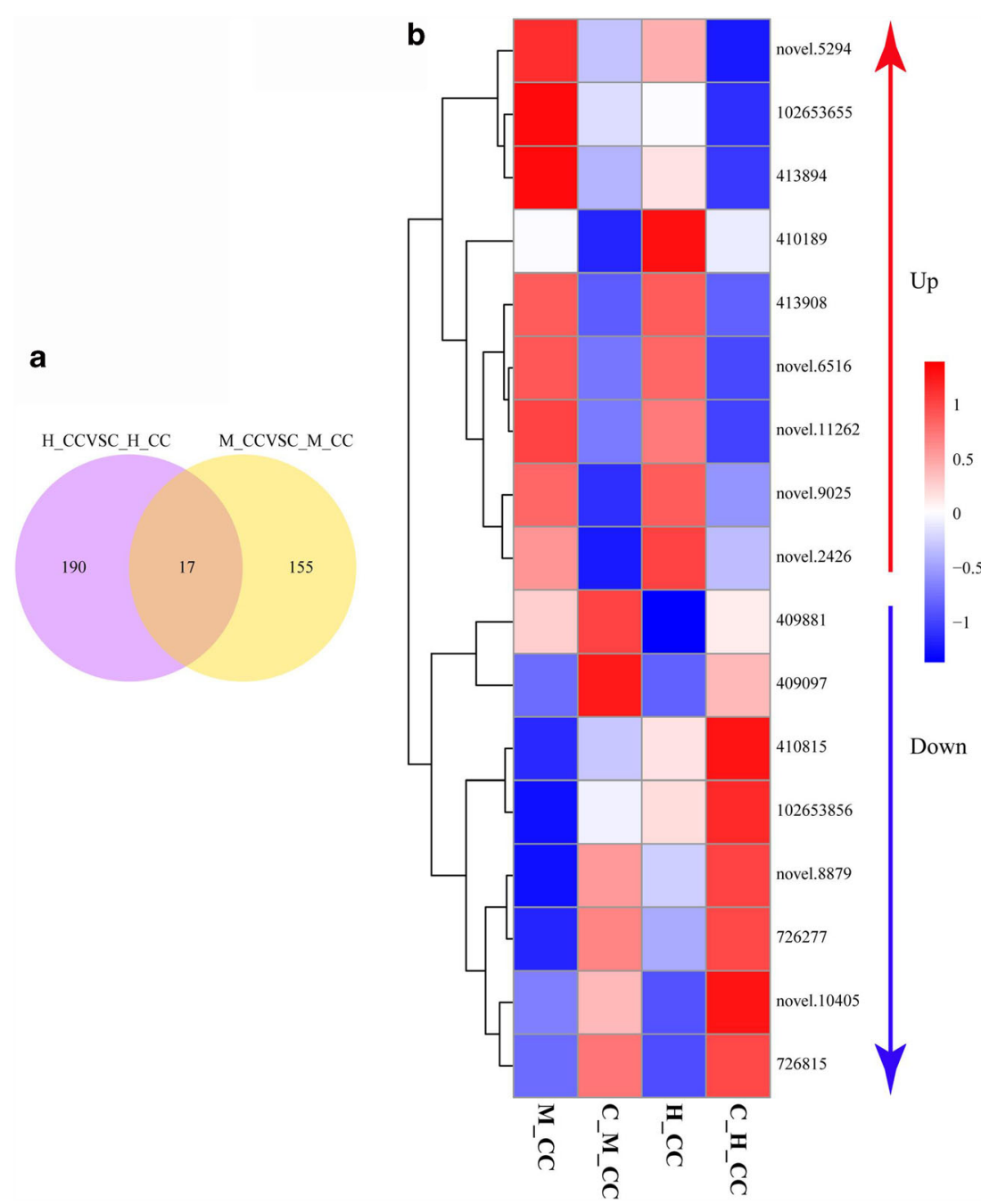

Figure 2. Common DEGs in the midguts and hemolymph of resistant larvae exposed to A. apis infection. a Venn diagram of unique DEGs in the midguts and hemolymph of resistant larvae exposed to A. apis. b Heatmap of 17 common DEGs in the midguts and hemolymph of resistant larvae exposed to A. apis infection.

3.5. Common DEGs in the midguts of the resistant and susceptible larvae exposed to $A$. apis

A total of 126 DEGs were shared in the midguts of the resistant and susceptible larvae exposed to A. apis (Figure 1a and Table S6). After the larvae were challenged with $A$. apis, defensin 1 was highly expressed with FPKM $=1046.7$ $(\sim 19$ fold $)$ and FPKM $=208.4(\sim 13$ fold $)$ in the midguts of the resistant M_CC and susceptible
M_TT larvae, respectively. Hymenoptaecin was extremely upregulated with FPKM = 3220.1 $(\sim 13$ fold) and FPKM $=925.3$ ( $\sim 3$ fold) in the midguts of the resistant and susceptible larvae, respectively (Figure 3). Many other genes, including protein toll, receptor-type guanylate cyclase gcy-4, probable cytochrome P450 6a14, vitellogenin, cytochrome P450 6k1, and insulin-like peptide receptor, were also upregulated when they were stimulated with A. apis . Among the 126 DEGs, only 4 genes were differentially expressed between the 


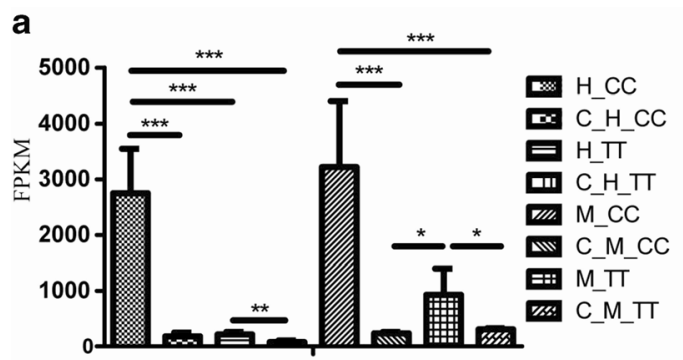

hymenoptaecin $(406142)$

C

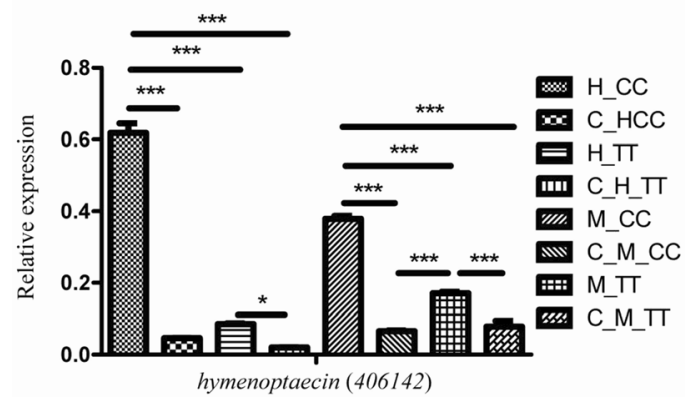

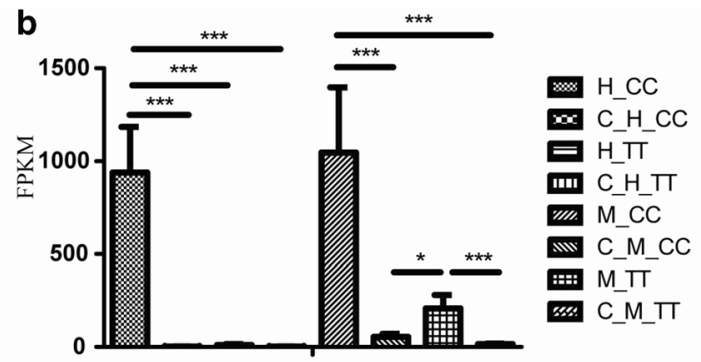

defensin I (406143)

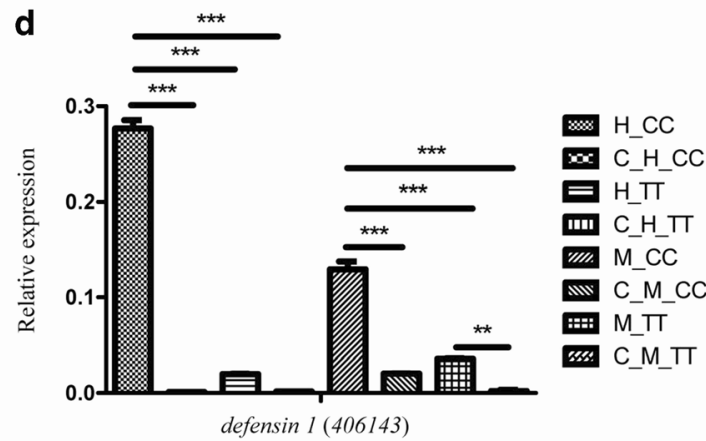

Figure 3. Hymenoptaecin and defensin 1 gene expressions by FPKM and qRT-PCR. a and $\mathbf{b}$ The FPKM values of expression profiles in the midguts and hemolymph of resistant/susceptible larvae exposed to A. apis, respectively. c and $\mathbf{d}$ The qRT-PCR values of expression profiles in the midguts and hemolymph of resistant/susceptible larvae exposed to A. apis, respectively. H_CC represents the hemolymph of the $\mathrm{C} / \mathrm{C}$ larvae from the treatment group; C_H_CC represents the hemolymph of the C/C larvae from the control group; H_TT: represents the hemolymph of the T/T larvae from the treatment group; C_H_TT: represents the hemolymph of the T/T larvae from the control group; M_CC: represents the midguts of the $\bar{C} / \bar{C}$ larvae from the treatment group; C_M_CC: represents the midguts of the $\mathrm{C} / \mathrm{C}$ larvae from the control group; $\mathrm{M}_{-} \mathrm{TT}$ : represents the midguts of the T/T larvae from the treatment group; C_M_TT: represents the midguts of the T/T larvae from the control group.

midguts of the resistant individuals M_CC and these of the susceptible individuals M_TT (Figure $4 \mathrm{a}$ and Table S7). Two genes, receptortype guanylate cyclase gcy-4 and uncharacterized LOC100577819, were upregulated in the midguts when the larvae were exposed to A. apis. Their expression was also upregulated in the resistant individuals M_CC compared with that in the susceptible individuals M_TT.

\subsection{Common DEGs in the hemolymph of the resistant and susceptible larvae exposed to A. apis}

Eighty-two genes exhibited the same expression differences in the hemolymph of resistant and susceptible larvae exposed to
A. apis (Figure 1c and Table S8). Among them, five genes were differentially expressed in the hemolymph of the resistant individuals H_CC and the susceptible individuals H_TT (Figure 4b and Table S9). Moreover, four genes, hymenoptaecin, insulin-like peptide receptor, and two novel genes, were upregulated in hemolymph when the larvae $\left(\mathrm{C}_{-} \mathrm{H} \_\mathrm{CC}\right.$ and C_H_TT) were exposed to A. apis. Their expression was also upregulated in resistant individuals H_CC compared with susceptible individuals H_TT. Surprisingly, the gene hymenoptaecin was extremely expressed $(\mathrm{FPKM}=2749.1)$ in the hemolymph of H_CC compared with that in the individuals of C_H_CC (FPKM = 181.4), H_TT (FPKM = $215)$, and C_H_TT $(\mathrm{FPKM}=1 \overline{8} 1.4)$. 

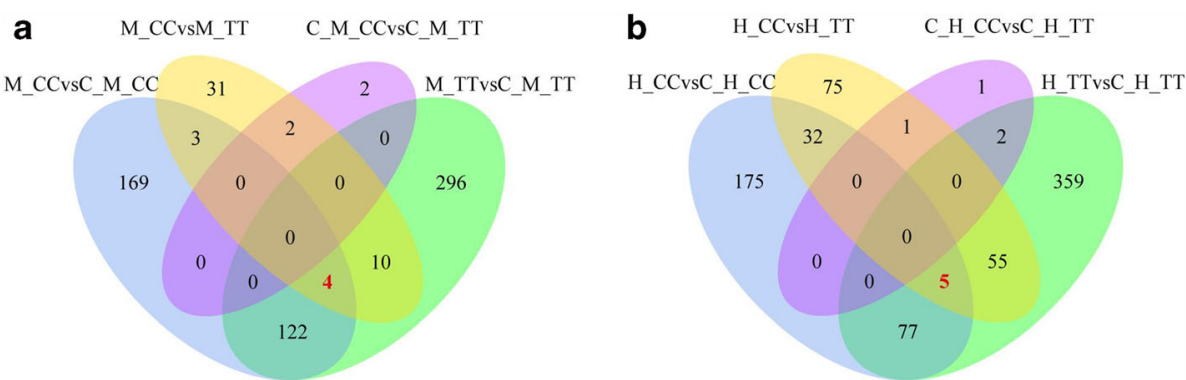

Figure 4. Venn diagram of DEGs in midguts and hemolymph. a Venn diagram of DEGs in the midguts of resistant and susceptible larvae. b Venn diagram of DEGs in the hemolymph of resistant and susceptible larvae.

\subsection{Common DEGs in the midguts and hemolymph of larvae exposed to A. apis}

A total of 126 common DEGs existed in the midguts of larvae M_CC and M_TT, and 82 common DEGs in the hemolymph of larvae $\mathrm{H}_{-} \mathrm{CC}$ and H_TT compared with corresponding groups (Figure 5). Among them, five genes, including hymenoptaecin, ataxin-2 homolog, insulin-like peptide receptor, protein G12-like, and a novel gene, were common DEGs in the midguts and hemolymph of the larvae exposed to A. apis (Table S10). The first three genes were upregulated in the midguts and hemolymph of the individuals inoculated with $A$. apis compared with those of the control group. The last two genes were downregulated. Specifically, hymenoptaecin was heavily expressed in the midguts of $\mathrm{M}$ _CC $(\mathrm{FPKM}=3220.1)$ and in the hemolymph of H_CC $(\mathrm{FPKM}=2749.1)$. Hymenoptaecin was also significantly upregulated in the hemolymph of H_CC compared with that in the hemolymph of H_TT $($ FPKM = 215). Therefore, larvae, especially resistant individuals, could rapidly respond to A. apis by increasing the expression of hymenoptaecin in the midguts and hemolymph.

\section{DISCUSSION}

In this study, resistant and susceptible larvae to A. apis according to the genotype of SNP C2587245T were initially determined. RNA-seq were then conducted to analyze the transcriptomes of the midguts and hemolymph in the resistant individuals. It was found that the DEGs in the midguts and hemolymph were predicted to have functions consistent with the resistant mechanisms of honey bees against $A$. apis.

When 2- to 4-day-old larvae are inadvertently fed spores of $A$. apis by adult bees via food provisions, the spores were ingested into midgut, which provided an anaerobic environment for spore germination, and then hyphae penetrate the gut epithelium and grow into the surrounding tissues, including hemolymph, killing the honeybees (Evison 2015). During infection by A. apis, host immune responses to fungi were activated. Compared with susceptible individuals, there were 172 specific DEGs in the midguts of the resistant larvae with A. apis infection, while 207 unique DEGs in the hemolymph (Figure 1), implying that these gene may play a critical role in the midguts/hemolymph of resistant larvae for defense against infection. Among them, nine genes, including mucin-19, yellow-e3, cytochrome P450, 5'-nucleotidase domaincontaining protein 3 , and five novel genes, were upregulated in the midguts and hemolymph of the resistant larvae. Insect chitinases belong to glycoside hydrolase family 18 , which possesses a highly conserved Glyco_18 catalytic domain. Glyco_18 chitinases exhibit antifungal activities, including reductions in hyphal diameter, hyphal branching, and conidia size (Fung et al. 2002; Khondkar Ehteshamul et al. 2006). A previous study showed that the expression of glycosyl hydrolase 18-like protein (insect chitinase-like protein) with the characteristic Glyco_18 catalytic domain was upregulated at the 24 -h post-infection 

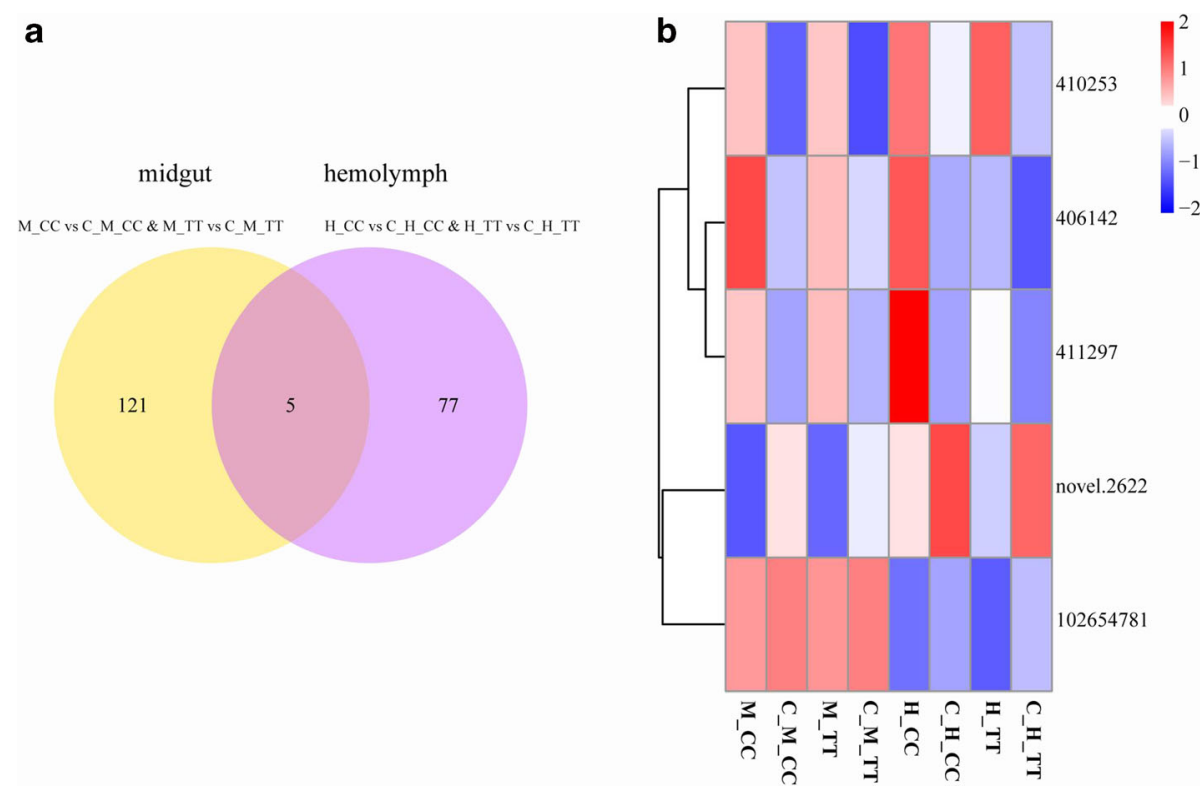

Figure 5. Common DEGs in the midguts and hemolymph of larvae exposed to A. apis infection. a Venn diagram of unique DEGs in the midguts and hemolymph of larvae exposed to A. apis. b Heatmap of five common DEGs in the midguts and hemolymph of larvae exposed to A. apis infection.

of $A$. apis (Cornman et al. 2012). In our study, mucin-19 containing the canonical domain of glycosyl hydrolase family 18 (Glyco_hydro_18) was upregulated in the midguts and hemolymph of the resistant individuals upon A. apis infection, suggesting that this gene might enhance the antifungal activity in response to chalkbrood disease. Melanization is an immune effector mechanism involved in the killing of bacteria, fungi, and parasites (Nuti et al. 2017). This process is associated with insect yellow gene family. Yellow gene determines the degree and pattern of melanization in Drosophila and Bombyx mori (Wittkopp et al. 2002; Futahashi et al. 2008). Previous study reported that yellow-f and yellow-f2 were involved in the melanization pathway, acting as dopachrome-conversion enzymes in Drosophila (Wu et al. 2018). In our study, yellow-e3 was upregulated in the midguts and hemolymph of the resistant individuals exposed to A. apis . This gene also belongs to insect yellow family containing the major royal jelly protein domain, suggesting that it might be involved in melanization to combat fungal infection. In insects, cytochrome P450 (CYP) participates in detoxifying insecticides and shows a close association with metabolic resistance. CYP2C19 genetic polymorphisms are correlated with invasive fungal infections in human disease (Lamoureux et al. 2016; Miao et al. 2019), suggesting that this gene may be critical for fungal resistance. Therefore, the significantly upregulated cytochrome P450 6A1 in the midguts and hemolymph of the resistant larvae challenged with $A$. apis suggested that cytochrome P450 6A1 might modulate fungal resistance.

AMPs are the first line of defense to combat invasive microbial pathogens in insects. Six honey bee AMPs, including abaecin, apidaecin, defensin-1, defensin-2, apsimin, and hymenoptaecin, have been identified in A. mellifera (Evans 2006). According to Figure 3, hymenoptaecin and defensin 1 were greatly upregulated in the midguts once the resistant and susceptible larvae were challenged with A. apis. The expression trend of hymenoptaecin gene in hemolymph was almost the same as that in midguts, whereas the 
expression of defensin 1 was strongly induced $(\mathrm{FPKM}=940$, approximately 500 fold $)$ in the hemolymph of the resistant individuals upon A. apis infection. However, the expression of defensin 1 was extremely low and did not significantly differ from that in the hemolymph of the susceptible larvae (H_TT and C_H_TT) (Figure 3 ). In addition, protein toll was upregulated in the midguts of the resistant and susceptible larvae when they were exposed to A. apis. Hymenoptaecin and defensin-1 were regulated solely by Imd and Toll pathways, respectively (Lourenço et al. 2013). Therefore, honey bee A. apis might trigger the Toll and Imd pathways in the midguts and activate the Imd pathway in the hemolymph of susceptible individuals, whereas Imd and Toll pathways might be activated in the hemolymph of resistant individuals. Antimicrobial resistance has posed a serious threat to global public health. AMPs have a broad spectrum of antimicrobial activity against microbes and can avoid emergence of resistance (Nuti et al. 2017). The use of AMPs also decreases the risk of chemical residues in honey and other hive products. Therefore, AMPs (hymenoptaecin and defensin-1) may be potential therapeutic agents for chalkbrood. In future studies, we may express hymenoptaecin and defensin-1 in vitro and determine their therapeutic efficacy on chalkbrood in honey bees.

The overall health status of larvae is directly correlated with their nutritional status. A tradeoff also occurs between immune stimulation and storage protein gene expression, such as vitellogenin (Lourenço et al. 2010). Currently, we observed that the expression of vitellogenin was upregulated in the midguts of the resistant and susceptible larvae exposed to A. apis. Insulin signaling pathway is nutrition sensitive, and it coordinates body and organ growth. This pathway is stimulated in well-fed Drosophila larvae but is suppressed under starvation (Julien et al. 2008; Charles et al. 2009). Here, insulin-like peptide receptor was upregulated in the midguts and hemolymph of the resistant and susceptible larvae exposed to A. apis, with a higher expression ( 3-fold, Table S10) in the hemolymph of the resistant individuals (H_CC) than that of susceptible individuals (H_TT). The body size of the resistant larvae exposed to A. apis was slightly larger than that of the susceptible groups inoculated with $A$. apis. We speculated that resistant larvae could improve nutrition storage and enhance the insulin signaling pathway to increase honey bees' resistance to chalkbrood disease.

This study presented a transcriptome analysis of resistant and susceptible honey bee larvae challenged with Ascosphaera apis, and identified DEGs in the midguts and hemolymph of infected individuals, which provided valuable information not only for better understanding the resistant mechanisms for $A$. apis infection, but also for selective breeding of chalkbroodresistant bee stocks. Meanwhile, it will give us an insight into the control of fungal diseases in hymenopterans.

\section{CONCLUSION}

In this study, 6-day-old larvae fed with an artificial diet were sequenced to determine the resistant and susceptible larvae for chalkbrood using SNP C2587245T. We comprehensively compared the gene expression in the midguts and hemolymph of the resistant and susceptible larvae through RNA-seq. The results indicated that 172 and 207 genes were specially and differentially expressed in the midguts and hemolymph of the resistant larvae infected with $A$. apis, respectively. Some DEGs were observed in the midguts and/or hemolymph. The data resources of the transcriptome suggested that the larvae might be resistant to chalkbrood disease by activating immune signaling pathways, melanization pathway, and cytochrome P450 genes and by improving nutritional status. AMP defensin 1 was particularly upregulated (approximately 500 fold) in the hemolymph of the resistant individuals upon $A$. apis infection. However, AMP defensin 1 had low or no significantly 
different expression in the hemolymph of the susceptible larvae (H_TT and C_H_TT). Consequently, applying AMPs might be a promising therapeutic strategy to control chalkbrood and the severe antimicrobial resistance.

\section{ACKNOWLEDGEMENTS}

This work was supported by the earmarked fund for Modern Agro-industry Technology Research System (NO. CARS-44-KXJ4), the Natural Science Foundation of Fujian Province, China (NO.2018J05043), and the scientific research program funded by the Fujian Provincial Department of Education for Provincial University (NO. JK2017014).

\section{AUTHORS' CONTRIBUTIONS}

HYN and SKS conceived this research and designed experiments; HYN, XYW, DLY, ZGL, and SKS participated in the design and interpretation of the data; XYW, SPX, YG, and YNZ performed experiments and analysis; HYN, YL, and SKS wrote the paper and participated in the revisions of it. All authors read and approved the final manuscript.

\section{COMPLIANCE WITH ETHICAL STANDARDS}

Conflict of interest The authors declare that they have no potential conflict of interest in relation to the study in this paper.

Changements dans l'expression génétique de la résistance au couvain plâtré chez les larves d' Apis mellifera infectées par Ascosphaera apis.

résistance au couvain plâtré / ARN-seq / intestins moyens / hémolymphe / peptides antimicrobiens.

Änderungen in der Genexpression bei kalkbrutresistenten Apis mellifera Larven nach Infektion mit Ascosphaera apis .

Kalkbrutresistenz / RNA-seq / Mitteldarm / Hämolymphe / antimikrobielle Peptide.

\section{REFERENCES}

Aronstein, K., Holloway, B. (2013) Honey bee fungal pathogen, Ascosphaera apis; current understanding of host-pathogen interactions and host mechanisms of resistance, In: Méndez-Vilas A. (Ed.), Microbial Pathogens and Strategies for Combating Them-Science, Technology and Education, Formatex Research Center, Spain, pp. 402-410

Aronstein, K.A., Murray, K.D. (2010) Chalkbrood disease in honey bees. J. Invertebr. Pathol. 103, (103 Suppl 1): S20-S29

Aronstein, K.A., Murray, K.D., de León, J.H., Qin, X., Weinstock, G.M. (2007) High mobility group (HMGbox) genes in the honeybee fungal pathogen Ascosphaera apis . Mycologia 99, (4): 553-561

Aronstein, K.A., Murray, K.D., Saldivar, E. (2010) Transcriptional responses in honey bee larvae infected with chalkbrood fungus. BMC Genomics 11, (1): 391

Chan, Q.W.T., Melathopoulos, A.P., Pernal, S.F., Foster, L.J. (2009) The innate immune and systemic response in honey bees to a bacterial pathogen, Paenibacillus larvae. BMC Genomics 10, 387

Charles, G., Rulifson, E.J., Pierre, L. (2009) Remote control of insulin secretion by fat cells in Drosophila. Cell Metab. 10, (3): 199-207

Chen, D., Guo, R., Xu, X., Xiong, C., Liang, Q., et al. (2017) Uncovering the immune responses of Apis mellifera ligustica larval gut to Ascosphaera apis infection utilizing transcriptome sequencing. Gene 621, 40-50

Chen, D., Guo, R., Xiong, C., Zheng, Y., Hou, C., et al. (2018) Morphological and molecular identification of chalkbrood disease pathogen Ascosphaera apis in Apis cerana cerana. J. Apic. Res. 57 (4)

Cornman, R.S., Bennett, A.K., Murray, K.D., Evans, J.D., Elsik, C.G., et al. (2012) Transcriptome analysis of the honey bee fungal pathogen, Ascosphaera apis: implications for host pathogenesis. BMC Genomics, 13, 285-285

Dillies, M.A., Rau, A., Aubert, J., Hennequet-Antier, C., Jeanmougin, M., et al. (2013) A comprehensive evaluation of normalization methods for Illumina highthroughput RNA sequencing data analysis. Brief. Bioinformatics 14, (6): 671-683

Evans, J.D. (2006) Beepath: An ordered quantitative-PCR array for exploring honey bee immunity and disease. J. Invertebr. Pathol., 93 (2): 135-139

Evison, S.E.F. (2015) Chalkbrood: epidemiological perspectives from the host-parasite relationship. Curr. Opin. Insect Sci. 10, 65-70

Flores, J.M., Gutiérrez, I., Espejo, R. (2005) The role of pollen in chalkbrood disease in Apis mellifera : transmission and predisposing conditions. Mycologia 97, (6): 1171-1176 
Fung, K.L., Zhao, K.J., He, Z.M., Chye, M.L. (2002) Tobacco-expressed Brassica juncea chitinase BjCHI1 shows antifungal activity in vitro. Plant Mol. Biol. 50, (2): 283-294

Futahashi, R., Sato, J., Meng, Y., Okamoto, S., Daimon, T., et al. (2008) yellow and ebony are the responsible genes for the larval color mutants of the silkworm Bombyx mori. Genetics 180, (4): 1995-2005

Guo, R., Chen, D., Xiong, C., Hou, C., Zheng, Y., et al. (2018a) Identification of long non-coding RNAs in the chalkbrood disease pathogen Ascospheara apis. J. Invertebr. Pathol. 156, 1-5

Guo, R., Wang, H., Chen, H., Xiong, C., Zheng, Y., et al. (2018b) Identification of Ascosphaera apis microRNA and investigation of its regulation networks. Acta Microbiol. Sin. 58, (6): 1077-1089

Jensen, A.B., Pedersen, B.V., Eilenberg, J. (2009) Differential susceptibility across honey bee colonies in larval chalkbrood resistance. Apidologie 40, (5): 524-534

Jensen, A.B., Aronstein, K., Flores, J.M., Vojvodic, S., Palacio, M.A., et al. (2013) Standard methods for fungal brood disease research. J. Apic. Res. 52, (1): 79-88

Julien, C., Sophie, R., Sophie, P., Thomas, R., Jacques, M., et al. (2008) A nutrient sensor mechanism controls Drosophila growth. Cell 114, (6): 739-749

Khondkar Ehteshamul, K., Daizo, H., Katsuhiro, W., Daizo, K. (2006) Purification and characterization of a novel isozyme of chitinase from Bombyx mori. Biosci. Biotechnol. Biochem. 70, (1): 252-262

Kim, T., Kim, Y.J. (2005) Overview of innate immunity in Drosophila. J. Biochem. Mol. Biol. 38, (2): 121-127

Lamoureux, F., Duflot, T., Woillard, J.B., Metsu, D., Pereira, T., et al. (2016) Impact of CYP2C19 genetic polymorphisms on voriconazole dosing and exposure in adult patients with invasive fungal infections. Int. J. Antimicrob. Agents 47, (2): 124-131

Li, Z., You, X., Wang, L., Yan, Z., Zhou, Z. (2018) Spore morphology and ultrastructure of an Ascosphaera apis strain from the honeybees (Apis mellifera) in southwest China. Mycologia 1-14

Liao, Y., Smyth, G.K., Shi, W. (2014) featureCounts: an efficient general purpose program for assigning sequence reads to genomic features. Bioinformatics 30, (7): 923-930

Liu, Y., Yan, L., Li, Z., Huang, W.F., Pokhrel, S., et al. (2016) Larva-mediated chalkbrood resistanceassociated single nucleotide polymorphism markers in the honey bee Apis mellifera. Insect Mol. Biol. 25, (3): 239-250

Lourenço, A.P., Martins, J.R., Bitondi, M.M., Simões, Z.L. (2010) Trade-off between immune stimulation and expression of storage protein genes. Arch. Insect Biochem. Physiol. 71, (2): 70-87
Lourenço, A.P., Guidugli-Lazzarini, K.R., Freitas, F.C.P., Bitondi, M.M.G., Simões, Z.L.P. (2013) Bacterial infection activates the immune system response and dysregulates microRNA expression in honey bees. Insect Biochem. Mol. Biol. 43, (5): 474-482

Lourenço, A.P., Florecki, M.M., Zlp, S., Evans, J.D. (2018) Silencing of Apis mellifera dorsal genes reveals their role in expression of the antimicrobial peptide defensin-1. Insect Mol. Biol. 1-13

Miao, Q., Tang, J.T., Van Gelder, T., Li, Y.M., Bai, Y.J., et al. (2019) Correlation of CYP2C19 genotype with plasma voriconazole exposure in South-western Chinese Han patients with invasive fungal infections. Medicine 98, (3): e14137

Mortazavi, A., Williams, B.A., McCue, K., Schaeffer, L., Wold, B. (2008) Mapping and quantifying mammalian transcriptomes by RNA-Seq. Nat. Methods 5, (7): 621-628

Nie, H., Liu, C., Cheng, T., Li, Q., Wu, Y., et al. (2014) Transcriptome analysis of integument differentially expressed genes in the pigment mutant (quail) during molting of silkworm, Bombyx mori. Plos One 9, (4): e94185

Nie, H., Xu, S., Xie, C., Geng, H., Zhao, Y., et al. (2018) Comparative transcriptome analysis of Apis mellifera antennae of workers performing different tasks. Mol. Genet. Genomics 293, (1): 237-248

Nuti, R., Goud, N.S., Saraswati, A.P., Alvala, R., Alvala, M. (2017) Antimicrobial peptides: a promising therapeutic strategy in tackling antimicrobial resistance. Curr. Med. Chem. 24, (38): 4303

Pertea, M., Pertea, G.M., Antonescu, C.M., Chang, T.C., Mendell, J.T., et al. (2015) StringTie enables improved reconstruction of a transcriptome from RNA-seq reads. Nat. Biotechnol. 33, (3): 290-295

Qin, X., Evans, J.D., Aronstein, K.A., Murray, K.D., Weinstock, G.M. (2006) Genome sequences of the honey bee pathogens Paenibacillus larvae and Ascosphaera apis. Insect Mol. Biol. 15, (5): 715-718

Skou, J.P. (1988) More details in support of the class Ascosphaeromycetes. Mycotaxon 31, (1): 191-198

Theantana, T., Chantawannakul, P. (2008) Protease and $\beta$ $\mathrm{N}$-acetylglucosaminidase of honey bee chalkbrood pathogen Ascosphaera apis. J. Apic. Res. 47, (1): 68-76

Vierstraete, E., Cerstiaens, A., Baggerman, G., Van den Bergh, G., De Loof, A., et al. (2003) Proteomics in Drosophila melanogaster: first 2D database of larval hemolymph proteins. Biochem. Biophys. Res. Commun. 304, (4): 831-838

Vojvodic, S., Jensen, A.B., James, R.R., Boomsma, J.J., Eilenberg, J. (2011) Temperature dependent virulence of obligate and facultative fungal pathogens of honeybee brood. Vet. Microbiol. 149, (1-2): 200-205 
Wittkopp, P.J., True, J.R., Carroll, S.B. (2002) Reciprocal functions of the Drosophila yellow and ebony proteins in the development and evolution of pigment patterns. Development 129, (8): 1849-1858

Wu, Q., Patočka, J., Kuča, K. (2018) Insect antimicrobial peptides, a mini review. Toxins 10, (11): 461

Zhang, N., Zhang, H.J., Zhao, B., Sun, Q.Q., Cao, Y.Y., et al. (2013) The RNA-seq approach to discriminate gene expression profiles in response to melatonin on cucumber lateral root formation. J. Pineal Res. 56, (1): $39-50$

Publisher's note Springer Nature remains neutral with regard to jurisdictional claims in published maps and institutional affiliations. 